\title{
UNA MIRADA CRÍTICA A LA FUNCIÓN ASESORA DESDE LOS SERVICIOS DE APOYO EXTERNO. RECORRIDO Y PROSPECTIVA
}

\section{A CRITICAL LOOK TO ADVISORY FUNCTION FROM THE SUPPORT SERVICES. TOUR AND PROSPECTIVE}

Jesús Domingo SEGOVIA* Víctor Hernández RIVERO**

\begin{abstract}
Resumo: Diante da mudança em educação, professores e escolas se vêem submetidos a uma alavanche de novos desafios, responsabilidades e exigências. Estes podem atuar tanto com elementos dinamizadores de autonomia curricular e capacidade profissional, ou como escudo ou véu que oculta - em projetos artificiosos, instigados e inspirados externamente - o que realmente ocorre e é importante nas salas de aula. Não vale qualquer mudança, o essencial da melhora em educação são as boas aprendizagens de todos os estudantes. E isso com bons professore e boas escolas, para o que é vital estimular e apoiar os processos orientados de melhora. Mas não se pode apoiar a mudança sem considerar as lições aprendidas no caminho. A partir dessa ótica, o artigo revisa o sistema de apoio, extrai conclusões e implicações sobre a prática profissional. E conclui oferecendo uma proposta viável do que deveria ser um bom assessoramento.
\end{abstract}

Palavras-chave: Mudança. Assessoramento. Melhora. apoio externo.

Abstract: Considering the change in education, teachers and schools are subjected to and avalanche of new challenges, responsibilities and demands. These issues can

* Universidad de Granada (España). E-mail: jdomingo@ugr.es

** Universidad de La Laguna (España). E-mail: vhernan@ull.es 
Una mirada crítica a la función asesora desde los servicios de apoyo externo...

act as revitalizing elements of curricular autonomy and professional ability, a shield or a veil that hides - in artful projects which are incited and inspired externally - what really happens and matters in the classroom. It is not worth any change, the importance of the improvement in education consist of the appropriate learning for all the students. This is due to good teachers and good schools, and because of them, it is vital to stimulate and support the guided processes of improvement. However, we cannot support changes without taking into account the learnt lesson in our path. From this perspective, the article revises the present system of support, draws conclusions and implications of the professional practice. Moreover, it ends offering a viable proposal about what an appropriate advice should be.

Keywords: Change in education. Advisement. Improvement. External support.

Profesores y escuelas se encuentran hoy más que nunca sometidos a una auténtica avalancha de nuevos retos y responsabilidades para los que no parecen estar preparados. Pese a ello, tienen posibilidades de cambio y desarrollo, siendo legión las experiencias que lo avalan y muestran comprometidas e interesantísimas innovaciones. Los centros educativos no son islas y necesitan de apoyo y acompañamiento para surcar por estos inciertos mares de la innovación y la mejora. Pero no vale cualquier apoyo ni a cualquier precio. Tampoco son cajas negras que producen los efectos deseados por el simple hecho de aplicarles unas determinadas recetas o unas mágicas fórmulas técnicas y administrativas. Luego, si los centros necesitan apoyo y no todo vale, ¿qué modelo de asesoría es el pertinente, cómo ha ido evolucionando esta idea y bajo qué principios y realidades actúa?

Ahora, con una larga experiencia acumulada, menos ingenuos y con nuevas perspectivas -como las propuestas desde la Asociación para el Desarrollo y Mejora de la Escuela (ADEME) (ESCUDERO; MORENO, 1992; DOMINGO, 2004) -, somos conscientes de que hay que resituar y repensar estos apoyos (HERNÁNDEZ; GUARRO, 2002; BONILLA, 2006; MONEREO; POZO, 2O07; COSTA, NETO; VENTURA, 2007; VV.AA., 2008), para garantizar el buen aprendizaje para todos en sociedades complejas (GARCÍA, 2008). 
En este contexto, este trabajo insiste en esta perspectiva de necesaria revisión crítica del proceso de evolución de los servicios de apoyo y su creciente reconstrucción identitaria hacia la función de asesoría crítica, y su complejo engarce en los personales procesos internos de (auto)revisión y mejora. Es fruto de un proyecto de investigación I+D, subvencionado por el Ministerio de Educación y Ciencia de España, que versa sobre la necesidad de redimensionar los servicios de apoyo. Y, desde ahí, recoge y sintetiza el saber pedagógico acumulado en este sentido, los sitúa en el contexto español y lo contrasta con buenas prácticas de asesoría. Para terminar apuntando algunos trazos gruesos de ese camino y concreta algunas pinceladas de "sentido común" para entender los servicios de apoyo y sobre las que convendría seguir indagando.

\section{CONSTRUCCIÓN CONTROVERTIDA DE LOS SERVICIOS DE APOYO EXTERNO}

La realidad actual de la escuela se hace cada vez más compleja y exigente. Cambiar el patrón de escolarizar por el de garantizar para todos el derecho a un buen aprendizaje, es un reto importante que no puede ser abordado desde viejos y obsoletos patrones. Se hace necesario afrontar la incertidumbre y la complejidad actual del día a día y con las nuevas situaciones y dificultades de nuestro tiempo y contexto (postmoderno, cambiante, plural, exigente, diverso...). Hay que superar antiguas rutinas y afrontar pertinente la toma de decisiones curriculares, para hacer viable el binomio equidad y calidad.

Se hace patente, pues, por parte de los investigadores como por el propio profesorado, las escuelas y administradores educativos, la necesidad de contar con ayuda para apoyar los esfuerzos del profesorado para aumentar la calidad de sus servicios y poner en práctica nuevas propuestas curriculares. Como dicen Stoll y Fink (1999), los centros educativos pueden desarrollar de manera autónoma capacidad para la innovación y la mejora de las experiencias educativas, pero 'no son islas' y, con toda probabilidad, si los apoyamos externamente lograrán desarrollarse y avanzar más eficazmente. Lo cierto es que la presencia de estos profesionales del asesoramiento trabajando junto al profesorado constituye un reactivo en la dinámica de renovación de los centros. Su papel como mediadores entre el conocimiento pedagógico y las prácticas docentes y las necesidades del profesorado los 
Una mirada crítica a la función asesora desde los servicios de apoyo externo...

convierte en un elemento indispensable en los procesos de formación, cambio y mejora escolar.

Así pues, cada vez es más habitual contar con estructuras y agentes encargados de ofrecer ayuda y asistencia a las escuelas y a los profesores. Nos referimos a lo que ya Louis et al. (1985) denominaran sistemas de apoyo externo. Es decir, al conjunto de individuos organizados, cuya meta y sentido es el asesoramiento y ayuda profesional de quienes ponen en práctica los procesos de enseñanza-aprendizaje, actuando desde estructuras externas a los propios establecimientos educativos, buscando su capacitación y mejora.

Desde que se presta interés por la mejora, se han venido experimentando una serie de maneras, de modelos, de servicios y de estructuras de apoyo, no siempre con el debido seguimiento y continuidad. Desde un modelo de servicios, se pensó que para cada problema o función era necesaria una estructura específica y especializada de apoyo.

Han transcurrido prácticamente treinta años desde que a finales de los 70 y principios de los 80 surgieran en España los primeros Servicios de Orientación Educativa y Vocacional, con funciones muy especializadas y dirigidas al diagnóstico, la educación especial y la orientación educativa. Y éstos han ido evolucionando hasta ir integrándose en otras estructuras más globales y cercanas a los centros educativos. Incluso han llegado a ser parte de los mismos, como los departamentos de orientación en los actuales institutos de Educación Secundaria. También han pasado veinticinco años desde el nacimiento en España -a mediados de los 80- de los Centros de Profesores (CEP/ CPR), inspirados en los 'Teachers Centers' británicos. Éstos vienen a cubrir las necesidades de formación permanente del profesorado. Y todo ello conviviendo con el servicio de Inspección Educativa, los diferentes especialistas (Educación Especial, Trabajadores Sociales, etc.) y otros programas institucionales que respondían a los grandes retos que las distintas Administraciones Educativas iban detectando (Bilingüísmo, Escuela Espacio de Paz, Ecoescuelas, Educación Compensatoria, Interculturalidad, Centros TIC, etc.). Cada uno actuaba a su ritmo y con sus prioridades, sin una visión sistémica, ni siquiera contextualizada a cada escuela. Lo que, evidentemente, tenía lagunas importantes y solapamientos evidentes.

Junto a esta idea de desarrollar e institucionalizar servicios con funciones de apoyo y asesoramiento en el sistema educativo, se ha 
vivido un cambio acelerado de leyes educativas y se ha ido desarrollando también y completando el mapa autonómico, que organizaba los servicios de apoyo en función de las prioridades de cada Comunidad Autónoma. Sin embargo, los sistemas de apoyo externo son estructuras organizativamente complejas que han ido estableciéndose y creciendo de manera poco sistemática y también diferenciada en las distintas Comunidades Autónomas que componen el Estado. El resultado es un amplio espectro de servicios y profesionales diversos que, desde diferentes enfoques y centros de interés intentan apoyar la mejora de la escuela. Desde sus orígenes tuvieron que cubrir un escenario controvertido y un espacio profesional sin mucha definición, al tiempo que se ha ido evolucionando en el concepto y función de asesoría sin demasiados consensos más allá de aparecer formalizada la función, sin especificar el rol o la orientación desde la que desarrollarla. Por lo que es habitual encontrar dificultades a la hora de su gestión y administración, así como en su estudio y análisis.

No se trata en este momento pues, de hacer una descripción de la panorámica, para extrapolar, sino de buscar una comprensión holística del conjunto, para identificar indicadores, circunstancias y enfoques, con sus luces y sombras en su desarrollo, para, fundamentalmente, trazar líneas gruesas de por dónde caminar como apoyo a la mejora de las escuelas, independientemente del servicio o programa del que se trate. Por ello decíamos, que el asesoramiento está emergiendo como un espacio intermedio en el que tenemos más claro qué es lo que no funciona y qué directrices parecen tener buen impacto para garantizar el desarrollo de procesos internos de mejora, que quién debe desempeñar estar función. Hablábamos así más de construcción controvertida de la identidad asesora y de rasgos problemáticos a tener en consideración y de redimensionar el asesoramiento y los roles o maneras de desempeñar la función asesora en función del contexto y los centros de interés, que de asesor o de describir funciones de servicios y profesionales (DOMINGO, 2004, p. 67; 183; ESCUDERO; MORENO, 1992, p. 231).

Son ya treinta años de lo que ha venido a llamarse asesoramiento institucional -esto es, asesoramiento organizado y estructurado - ofertado desde sistemas de apoyo a la escuela. Entre estos servicios tienen cabida una multitud de iniciativas y prácticas de apoyo entre las que se encuentran las de asesoramiento, entremezclándose y confundiéndose a veces como prácticas de orientación, a veces como formación, 
Una mirada crítica a la función asesora desde los servicios de apoyo externo...

o simplemente como actividades de asistencia y colaboración entre profesionales para la resolución de necesidades y problemas en el seno de nuestras escuelas. Y tal ha sido su diversidad y tipología, su riqueza y amplitud, que se han invertido no pocos esfuerzos, debates y ríos de tinta para comprender y definir, clasificar y etiquetar un ingente y variopinto conjunto de prácticas que convenimos en llamar, de asesoramiento.

En la medida que se clarificasen dichos servicios en torno a un mismo esquema o perfil que sirviese de marco de referencia, que unificase, estructurase y facilitase el lenguaje sería posible mejorar la comunicación y discusión sobre el sistema. Eso fue permitiendo la planificación, coordinación y diseño del mapa de servicios y actividades de apoyo que se ofertan e ir identificando cuándo se producen vacíos y solapamientos. Luego proliferaron estudios en el contexto español (ESCUDERO; MORENO, 1992; MARCELO, 1997; HERNÁNDEZ; GUARRO, 2002; MARTÍN, 1999; NIETO; BOTÍAS, 2000) sobre estas estructuras, funciones, enfoques, permitieron un diagnóstico de la situación e identificaron toda una serie de problemas que venían afectando a dichas estructuras en su funcionamiento y organización que merecen la pena ser analizados. Lo que benefició la comprensión y reflexión sobre el fenómeno, barajar diferentes posibilidades en su configuración $\mathrm{y}$, en consecuencia, la posibilidad de cambiarlo y mejorarlo.

Sus hallazgos apuntan a que aunque formalmente los servicios de apoyo en las distintas zonas eran iguales, en la práctica existían grandes diferencias en el trabajo que desarrollaban. Algunos miembros que ocupaban los mismos puestos realizaban funciones distintas. Aunque la estructura de un servicio era similar a la de otro servicio, en la práctica realizaban su trabajo de formas diferentes. Encontraron que existía una gran diversidad y dispersión de prácticas.

En toda esta investigación se constataba también que el volumen de trabajo de los agentes había aumentado y no se habían tenido en cuenta las consecuencias. En las zonas en las que los equipos de apoyo contaban con menos asesores este problema era más grave, ya que existían pocos puestos para cubrir adecuadamente todas las áreas del currículo. Con frecuencia lo que ocurría es que se distribuían áreas de materias a asesores tanto si tenían experiencia en las mismas como si no. Como consecuencia directa, los asesores estaban sobrecargados de trabajo, siendo sus jornadas interminables.

Paralelamente, existía la necesidad de definir mucho más las tareas de los asesores. Una de las razones por las que los asesores 
se veían saturados de trabajo era la adición constante de nuevas funciones y programas sin que se restase ninguna de las que ya se llevaban a cabo. A lo que había que añadir el hecho de que los trabajos no estaban definidos con claridad. Tampoco había canales adecuados para evaluar su sus modelos de funcionamiento, funciones fundamenteles, etc.

Además, gran parte de la tarea de apoyo que realizaban los servicios de asesoramiento era improvisada. Es decir, una cantidad importante de tiempo y esfuerzo de su labor profesional se utilizaba en sacar adelante el trabajo según iba surgiendo día a día, sin apenas llevar a cabo planificación alguna. Esta improvisación era provocada en parte por la enorme presión bajo la que realizaban su trabajo, o bien por los efectos del lado oscuro del asesoramiento (tiranía de lo cotidiano, hacer lo que manda la tradición, burocratización, etc.).

Por otra parte, estos estudios concluían que la estructura y organización de muchos servicios de asesoramiento estaban anticuadas. Muchos sistemas de apoyo mantenían la estructura según el modelo establecido en la anterior reforma, aunque ésta ya no fuera idónea. Seguían instalados en funciones marginales del sistema o desde plateamiento técnicos obsoletos -en gran medida- para la marcha cotidiana del proceso de desarrollo institucional y de mejora de la educación en el día a día de clase.

Así mismo, existía una carencia importante en cuanto al asesoramiento, fundamentalmente por la falta de experiencia en este tipo de puesto de trabajo del personal contratado como asesor. Se necesitaba mejorar la dirección y coordinación de los sistemas de apoyo, sobre todo en un momento en el que el trabajo del servicio estaba cambiando y se hacía necesario un liderazgo que marcara la trayectoria. La falta de liderazgo llevó a una falta de estructura y a una debilidad organizativa en el servicio. Existía una tendencia a trabajar de manera aislada y a que hubiera poco trabajo en equipo.

La comunicación dentro de los propios servicios de asesoramiento, con los centros educativos y con la Administración no era buena, en parte como un efecto adicional de la falta de liderazgo. Los centros parecían estar poco seguros del papel de los asesores externos y sobre el mejor modo de utilizar sus servicios. También había una falta de comunicación dentro de los sistemas de apoyo y algunos miembros se sentían desconectados, impidiendo que todo el servicio trabajara para la consecución de unos mismos objetivos. 
Una mirada crítica a la función asesora desde los servicios de apoyo externo...

Por otro lado, no se tenía en cuenta la naturaleza especializada de la función asesora de manera que se presuponía que cualquiera con unos buenos antecedentes como profesor podía convertirse en un asesor sin recibir formación adicional específica. La formación que recibían los profesionales de los servicios de apoyo no era suficiente. Y necesitaban mayor apoyo administrativo y tecnológico que les liberara de las tareas más burocráticas, en las cuales se invertía mucho tiempo.

De acuerdo con Arencibia y Guarro (1999), cuestiones como la marginalidad, el celularismo, el elitismo, la redundancia, la sobrerregulación, la sobrecarga y heterogeneidad de funciones, la baja credibilidad, y el carácter generalmente impuesto del apoyo pueden ser consideradas como notas conflictivas en su configuración. Comentaremos, brevemente, cada una de ellas.

La marginalidad del rol del asesor constituye un factor de naturaleza estructural habitualmente problemático para el desempeño de las tareas en los sistemas de apoyo externo. La marginalidad puede ser entendida en distintos sentidos:

Los asesores experimentan su marginalidad en múltiples formas y direcciones: marginalidad respecto del contexto organizativo y, especialmente, del sistema de relaciones interpersonales del centro al que asesoran; marginalidad de los temas de que se ocupan en relación con los que ocupan y preocupan a los asesorados; marginalidad del conocimiento que dicen poseer y que han de transferir en relación con el que los asesorados demandan; en su caso, marginalidad también de los alumnos con los que trabajan directamente; $y$, sobre todo, la marginalidad fundamental que supone estar a mitad de camino entre dos culturas, la de la Administración y la de los centros y profesores, de modo que la Administración los trata como a docentes (o en algunos casos incluso peor), y los docentes tienden a percibirlos y tratarlos con el recelo y el desentendimiento con el que tratan a los representantes de la Administración. (MORENO, 1999, p. 251).

Esta característica que coloca a los agentes de apoyo entre espacios divergentes a distintos niveles, genera dificultades respecto del papel y actuación a desempeñar y requiere por su parte altas dosis de habilidad para hacerle frente y transformarla. Entre otras cuestiones, incide en la percepción que los propios asesores tienen de su labor, 
especialmente en relación con la satisfacción personal y profesional que este tipo de labores proporciona, condicionando los incentivos y recompensas que reporta, y en la percepción que tienen centros educativos y profesorado, mediatizando las relaciones interpersonales y las dinámicas de trabajo.

Otro de los problemas habituales en la evolución de estas estructuras en el seno de los sistemas educativos tiene que ver con el carácter celularista y divisionario con que se han gestado y, hoy por hoy, siguen funcionando. Según Nieto (1996, p. 220), "los modelos de apoyo imperantes han funcionado sobre la base de dos características esenciales: el celularismo y el elitismo".

Los sistemas de apoyo externo, constituidos por especialistas en ámbitos disciplinares diversos, habitualmente trabajando cada uno por su lado, han adoptado como patrón de relación el elitismo, es decir, separando productores y usuarios del conocimiento. Para Moreno (1999, p. 252), "la descoordinación entre las distintas fuentes de apoyo en nuestro sistema es proverbial. Entre ellas no solamente se ha venido produciendo ignorancia mutua y solapamiento de funciones, sino también, en muchos casos, una rivalidad manifiesta", lo que ha provocado un asesoramiento fragmentario, episódico y pasajero, sin una perspectiva global del contexto en el que ocurre, con pocas probabilidades para favorecer cambios reales y duraderos.

El celularismo, como fenómeno que afecta al asesoramiento se manifiesta en que cada sistema de apoyo y cada profesional tiende a proteger y a configurar su ámbito de influencia específico, diferenciándolo del de los demás, haciendo uso de un discurso propio, estableciendo instrumentos y procedimientos particulares.

Como consecuencia de ello, otro rasgo problemático tiene que ver con la redundancia, es decir, diferentes sujetos en un mismo contexto realizan la misma actividad por separado, prestando el mismo servicio a centros y profesores, adoptando en ocasiones el mismo rol y perfil profesional.

El problema de la redundancia se produce tanto por la descoordinación como por la rivalidad entre sistemas de apoyo, pero también por otros factores como la saturación del campo dada la cantidad de fuentes y modalidades de apoyo disponibles. Este hecho acarrea, como consecuencia, efectos no deseados como una imagen de despilfarro de recursos, de solapamiento indebido, de pérdida de tiempo y de infrautilización de recursos y materiales. 
Una mirada crítica a la función asesora desde los servicios de apoyo externo...

También afectan directamente al funcionamiento de los sistemas de apoyo externo es la sobrerregulación de funciones, estrategias y competencias, pues genera sobrecarga. En parte, la sobrerregulación está provocada por la falta de tradición y conocimiento de este tipo de labores, constituyéndose en un intento de las Administraciones educativas por ejercer un control estrecho de estos profesionales. Aunque entre sus causas también se encuentra el movimiento de descentralización administrativa del sistema educativo ya que con frecuencia las Administraciones regionales y/o locales que han asumido nuevas competencias, han tendido a reproducir las estructuras y modelos de gestión de la Administración central o estatal, generando todo un cuerpo de legislación y normativa muy específica al respecto, intentando delimitar y definir con precisión esta nueva función, pero no siempre en el sentido más interesante.

Por último, la configuración de los sistemas de apoyo externo como instrumentos al servicio de la política educativa de la Administración para difundir, promocionar y poner en marcha sus propuestas de reforma, más que como recursos al servicio de las escuelas, ha teñido de un carácter impuesto y contradictorio la oferta de ayuda y asesoramiento al profesorado y los centros. Una vez superadas las fases de desconocimiento y desconfianza, y están asentados los servicios, tampoco terminan de llegar al aula y el profesorado. Su acción, enmarcada en torno a programas externos y estelares, que más que promover iniciativas y mejoras internas han venido a maquillar las antiguas propuestas gerencialistas y externas que intentaban iluminar y domesticar a los centros, con las consecuencias obvias de esta distancia de concepciones, lenguajes, intereses, vehículos de acción...

\section{NUEVOS RETOS Y PROPUESTAS EMERGENTES EN LA CONFIGURACIÓN DEL ASESORAMIENTO COMO PLATAFORMA DE APOYO}

Son varios los indicadores de la evolución vivida en las últimas décadas respecto a la función de asesoramiento y los sistemas de apoyo como elementos estructurales clave en la mejora de los sistemas educativos. Por ello, existe una clara tendencia hacia la institucionalización, estabilización y mayor definición de estos servicios como consecuencia de una mejor conceptualización de la función y un mayor desarrollo organizativo de sus estructuras. Los sistemas de apoyo externo paulatinamente han ido perdiendo su carácter novedoso 
o experimental, transformándose en elementos permanentes en los sistemas educativos.

Se aprecia cada vez más un creciente grado de integración y coordinación de estas estructuras frente a la confusión, duplicación y derroche de recursos anterior. Aunque existe gran variedad de servicios de asesoramiento, se evoluciona hacia la configuración de grandes redes que integran y concentran a éstos, con una base política y filosófica definida, compartiendo valores y criterios de acción. Al mismo tiempo que se ha producido una expansión y especialización de los sistemas de apoyo, parejo al avance y evolución del propio sistema educativo. El incremento en su financiación y presupuestos, y el aumento en la cantidad y calidad de las demandas por parte de profesores y centros también indican esta tendencia.

Se va haciendo evidente una presión cada vez mayor hacia la rendición de cuentas y alcanzar unos estándares de calidad, lo que se ha traducido en evaluaciones, tanto internas como externas, también relativas a los propios servicios y programas, así como estudios e investigaciones sobre este tipo de fenómeno educativo, lo que está provocando un aumento en el conocimiento disponible al respecto.

Todos estos conflictos y problemas requieren para su resolución, mayor atención de la prestada y una mayor conjunción de esfuerzos e intereses, aunando perspectivas divergentes sobre el particular, articulando y configurando un modelo general sobre lo que ha de ser el asesoramiento a los centros educativos. En definitiva, la llegada de nuevos retos terminó por dinamitar las viejas estructuras, para demandar nuevas perspectivas.

En este contexto tan complejo que dibujamos, muchos profesionales han contribuido a construir un marco comprensivo que nos ha permitido ir atisbando referentes y caminos. Tanto desde el ámbito de la práctica, como desde la teoría y la investigación, nos han propuesto sus reflexiones y sus puntos de vista, sus experiencias y ejemplos, que ha hecho posible contar actualmente con un marco teórico y experiencia suficientes sobre este tipo de labores desde un marco coherente con una perspectiva de formación en centros y de (re)construcción interna de las escuelas como comunidades de aprendizaje (BOLÍVAR y otros, 2007; DOMINGO, 2004; BONILLA, 2006).

Así, hemos vivido el paso del modelo servicios a actuar dentro del modelo de programas (ampliamente elogiado desde la administración educativa). Pero aún este último modelo se muestra insuficiente y había que seguir integrando acciones y servicios en estructuras 
Una mirada crítica a la función asesora desde los servicios de apoyo externo...

más próximas, colaborativas y enraizadas en los propios proyectos educativos de centro. En la actualidad nos encontramos en un momento de redefinición, de desarrollo y búsqueda de claves y modelos conceptuales que clarifiquen de qué hablamos cuando nos referimos al asesoramiento en educación. Desde un punto de vista práctico, éste es un momento de reordenación y regulación, de adaptación de los recursos y sistemas de apoyo existentes a un nuevo sistema educativo.

En este contexto, la mejora del apoyo externo requiere -entre otras cuestiones- una formación y capacitación técnica específica para estos profesionales (ESCUDERO, VALLEJO; BOTÍAS, 2008). Contar con la adecuada formación para los servicios de apoyo permitiría la adquisición de conocimientos y estrategias, el desarrollo de una mayor competencia y capacidad de respuesta en estos agentes, equipos y sistemas de asesoramiento. Pero,

tales capacidades no se adquieren por decreto ni de manera espontánea. Es necesario disponer de formación sobre cómo se construye y se ejerce la colegialidad, cómo se crean relaciones de colaboración con los centros educativos, cómo se conquista la credibilidad del asesor y, por tanto, se transforma la marginalidad inicial e inevitable de su función y su figura. (MORENO, 1999, p. 257).

El funcionamiento adecuado de los sistemas de apoyo requiere, al tiempo, mayor autonomía. Frente a la excesiva regulación e intervención por parte de la Administración, conceder - como ya sucede con los centros educativos y el profesorado - una mayor independencia en su gestión a estas estructuras permitiría actuaciones más adaptadas a las necesidades reales de las escuelas, con criterios más flexibles y abiertos.

Rebajar la presión de la normativa sobre los sistemas de apoyo permitiría un margen de acción para construir las relaciones entre asesores y asesorados y potenciar la innovación. La desregulación evitaría que la relación entre las políticas de apoyo y las prácticas escolares se materializaran exclusivamente por medio de mecanismos formales, que con frecuencia constituyen un mero ejercicio simbólico.

No se trataría de cambiar estructuras para generar determinadas prácticas, sino más bien de intentar hacer posible que, desde la práctica y las demandas que ésta genera, se vayan configurando, 
consolidando y expandiendo ciertas estructuras de relación entre los agentes implicados. (NIETO, 1996, p. 230-231).

Como punto de referencia principal, el asesoramiento y apoyo externo ha de configurarse en torno a la idea de que el centro educativo constituye la unidad básica del cambio. Cada escuela interpreta y pone en práctica la innovación de una manera particular al resto, en base a su cultura institucional, por lo que la ayuda externa que reciba ha de considerarse adaptada a cada contexto y realidad local. Por lo tanto, esto quiere decir que el eje desde el que articular las funciones y estrategias del apoyo externo no puede situarse en las coordenadas de la Administración, en relación a sus prioridades e intereses, sino que debe ponerse al servicio de escuelas y comunidades educativas para potenciar su autonomía y mejora, adoptando planes o contratos diferenciados de trabajo conjunto.

Constituye una necesidad avanzar hacia la integración y consolidación de estas estructuras de apoyo -dada la cada vez mayor complejidad y diversidad de servicios y perfiles - con una política basada en la coordinación de actuaciones y planes entre agentes y sistemas de apoyo. La complejidad de los procesos de cambio requiere la conjunción de asesoramiento de tipo generalista y especializado al mismo tiempo, desde posiciones tanto internas como externas al centro, con criterios y principios de actuación compartidos.

Al mismo tiempo, sería deseable una mayor estabilidad y permanencia de estas estructuras, garantizando de cara al futuro la función de asesoramiento profesional en el ámbito educativo, fortaleciendo, mejorando e institucionalizando de manera consistente los sistemas de apoyo externo, lo que sin duda constituiría una forma de invertir en la calidad educativa.

Los agentes de apoyo en el seno de los sistemas educativos constituyen un valioso recurso que cuando es utilizado adecuadamente, permite a los centros y el profesorado acometer con ciertas garantías de éxito procesos de revisión e innovación de la práctica, procesos que como se sabe, conllevan riesgo, incertidumbre y altas dosis de resistencia, pero que constituyen la única vía válida para mejorar de manera efectiva la educación y la enseñanza.

Garantizar al profesorado que cuenta con suficiente apoyo para iniciar y desarrollar sus planes y proyectos educativos puede significar un importante estímulo y una invitación para que los equi- 
Una mirada crítica a la función asesora desde los servicios de apoyo externo...

pos docentes asuman su cuota de responsabilidad en el proceso de mejorar las prácticas de enseñanza y los resultados de aprendizaje del alumnado, para avanzar en la construcción de centros educativos comprometidos con su entorno y su realidad cercana.

Por último, el desarrollo del currículum ha de constituirse en el contenido prioritario del asesoramiento externo. El apoyo a la mejora escolar requiere focalizar su mayor intensidad sobre los temas y cuestiones nucleares del sistema escolar, esto es, el currículum, la enseñanza y el aprendizaje. Como bien dicen Nieto y Botías (2000), uno de los retos planteados actualmente es lograr que los sistemas de apoyo puedan incidir en ámbitos fundamentales de la escuela como el currículum, los procesos organizativos del centro y los procesos de enseñanza y aprendizaje en el aula. Y que puedan hacerlo compartiendo la iniciativa y la responsabilidad con cada centro educativo.

En esta línea, los apoyos a la escuela deben ir dirigidos a aumentar la competencia del profesorado, no a suplantarla. Deben ir dirigidos a facilitar el desarrollo de los centros, colaborando en la renovación de su cultura, valores, procesos y prácticas. Deben ir dirigidos a lograr la mejora de la educación.

Y en todo ello, para no perder el norte, no se puede nublar el verdadero sentido del asesoramiento, que no es otro que ayudar a escuelas y profesores para que hagan efectiva la garantía del buen aprendizaje para todos los estudiantes. Desde asegurar unos mínimos imprescindibles de ciudadanía para todos, sin exclusiones, a buscar los máximos y mejores resultados posibles y deseables, sin olvidar la garantía de lo primero.

En suma, convendría debatir y consensuar mucho más el papel que los agentes y sistemas de apoyo y las funciones de ayuda y asesoramiento profesional han de jugar en los procesos de cambio y mejora educativa. Los sistemas de apoyo educativo constituyen un ámbito en el que se dan cita decisiones administrativas, políticas, profesionales, institucionales y educativas de gran calado e importancia para el conjunto del sistema educativo. A estas alturas parece claro que constituye un fenómeno que requiere mucha más atención que la prestada hasta ahora.

\section{ALGUNOS NORTES POR LOS QUE CAMINAR}

A grandes trazos la cuestión estriba en la necesidad de: 
1. Plantear nuevas maneras de asesoría (DOMINGO, 2003; 2004; 2005; BOLÍVAR y otros, 2007), que sitúen el asesoramiento desde una visión estratégica de proceso y como colegas críticos, que huyen de la posibilidad de hacerse infalibles y que actúan bajo los parámetros de: (a) "trabajar con" en lugar de "intervenir en"; (b) colaborar en desarrollar, no aplicar; (c) ser mediador y facilitador de procesos, en los que el propio grupo encuentra y asume sus autosoluciones/propuestas; y (d) equilibrar -como abogado del diablo y reflejo crítico de la realidad-las iniciativas innovadoras locales dentro de un marco objetivo comúnmente aceptado como válido. Todo ello resaltando la competencia y liderazgo de los propios equipos docentes en el proceso, para que se doten del "proceso de autorrevisión" como motor y herramienta de transformación que hagan posible el «buen aprendizaje de y para todos».

2. Refocalizar el trabajo para incidir en lo fundamental de manera significativa a cada escuela (DOMINGO, 2006):

- Ir de lo marginal (lo especial, específico, puntual, etc.) a lo nuclear (el aula, el currículum y la garantía de los aprendizajes imprescindibles para todos);

- Compartir un mismo lenguaje y una misma conceptualización del problema;

- Saltar de hablar de "problemas" (muy apegadas a casuísticas particulares) a hacerlo de "problemáticas" (más generalizables y objetivas);

- Ir conquistando y construyendo la competencia docente», su capacidad para encontrar sus autosoluciones, movilizando diversos recursos cognitivos (saberes, capacidades, informaciones, redes, etc.) en orden a actuar, con pertinencia y eficacia, y dotarlo de procesos reflexivos y de autoreevisión y mejora;

- Trabajar desde los programas y proyectos del centro y en el seno de sus equipos docentes y departamentos didácticos y no desde programas terapeutizados o especializados.

3. Junto a otras soluciones estratégico-funcionales desde el propio sistema, no es una revolusión, es un cambio desde la realidad y lo que tenemos, para dar un pasito más:

- Equilibrar, ajustar e integrar contextual y sistémicamente "autorrevisión y supervisión" con "innovación, asesoramiento y dotación". Es decir, (auto y hetero)evaluación formativa de centros en procesos de mejora 
Una mirada crítica a la función asesora desde los servicios de apoyo externo...

- Confluencia de servicios en cuanto a compartir, incluso solapar, la función de asesoramiento. Algunas propuestas superadoras de esta dicotomía podrían venir en líneas de acción que integren organizativa y funcional los servicios educativos de apoyo externo de supervisión y de asesoramiento. Tanto sea desde propuestas internas dentro de un programa marco de mejora, como desde la emergencia de equipos de zona educativa (comarcal o provincial) capaces de ofrecer una unidad básica de organización, seguimiento y apoyo a una red de centros.

- La coordinación e integración de los servicios de apoyo en una estructura global de zona educativa. Simplificar e integrar funcionalmente los servicios de apoyo externo dentro de estructuras globales y uniformes por comarcas o zonas educativas.

- Redimensionar algunas funciones de los equipos de apoyo, primando las de apoyo interno y asesoría crítico-colaborativa ante las burocrático-administrativas y las puramente especializadas.

- Creación de equipos de investigación-evaluación-asesoría, como técnicos, especialmente informados para prestar asesoría técnica sobre el funcionamiento y necesidades del Sistema a la Administración Educativa.

En definitiva, no se trata tanto de optar por un modelo u otro, sino de repensar la necesidad de buscar alternativas de integración y coherencia, sin dejar de lado apoyos y tareas particulares que también deben seguir promocionándose.

\section{REFERÊNCIAS}

ARENCIBIA, J. S.; GUARRO, A. Mejorar la escuela públic: una experiencia de asesoramiento a un centro con problemas de disciplina. Tenerife: Consejería de Educación del Gobierno de Canarias/CCPP, 1999.

BOLÍVAR, A, DOMINGO, J., ESCUDERO, J. M.; GONZÁLEZ, M. T.; GARCÍA, R. J. Asesoría pedagógica. Módulo I: El centro como contexto de formación. Madrid: Centro Nacional de Información y Comunicación Educativa (CENICE), 2007. Disponible en: http://formacion.cnice.mec.es/materiales/89/ $\mathrm{cd} / \mathrm{m} 1 /$ modulo1.pdf.

BONILLA, O. (Coord.). La asesoría a las escuelas: reflexiones para la mejora educativa y la formación continua de los maestros. México: SEP-PRONAP/ OEI/Cooperación Española, 2006.

COSTA, J. A., NETO-MENDES, A; VENTURA, A. (Orgs.). A assessoria na educação em debate. Aveiro: Universidade de Aveiro, 2007. 
DOMINGO, J. Repensar o assessoramento nos processos internos de desenvolvimento curricular. Pátio Revista Pedagógica. Disponible en: http://www. revistapatio.com.br/conteudo_exclusivo.aspx Acesso en: 11 jan. 2004.

DOMINGO, J. Las prácticas de asesoramiento a centros educativos: una revisión del modelo de proceso. Archivos Analíticos de Políticas Educativas (EPAA), Disponible en: http://epaa.asu.edu/epaa/v13n17/v13n17.pdf Acesso en: 13 ago.2007.

DOMINGO, J. Los departamentos de orientación en la mejora cualitativa de la educación secundaria. Revista de Educación, Disponible en: http://www. revistaeducacion.mec.es/e339/re339a07.pdf Acesso en: 20 jun. 2007.

DOMINGO, J. (Coord.). Asesoramiento al centro educativo: colaboración y cambio en la institución. México: SEP/Octaedro, 2004.

ESCUDERO, J. M.; MORENO, J. M.: El asesoramiento a centros educativos: estudio evaluativo de los equipos psicopedagógicos de la Comunidad de Madrid. Madrid: CECCAM, 1992.

ESCUDERO, J. M.; VALLEJO, M.; BOTÍAS, F. El asesoramiento en educación: ¿Podrían ser las competencias profesionales una contribución positiva? Profesorado, revista de currículum y formación del profesorado, Disponible en: http://www.ugr.es/local/recfpro/rev121ART2.pdf Acesso en: 20 abr. 2008.

GARCÍA, R. J. El asesoramiento escolar en sociedades complejas. Profesorado, revista de currículum y formación del profesorado, Disponible en: http:// www.ugr.es/local/recfpro/rev121ART1.pdf Acesso en: 20 abr. 2008

HERNÁNDEZ, V.; GUARRO, A. Asesoramiento y sistemas de apoyo a los centros educativos: estudio de los equipos pedagógicos de los Centros del Profesorado (CEP) de Canarias. La Laguna: DGOIE del Gobierno de Canarias, 2002.

LOUIS, K. S. et. al. External support systems for school improvement. In: VAN DEN BERG, R.; HAMEYER, U.; STOKKING, K. (Eds.). Dissemination reconsidered: the demands of implementation. Leuven: ACCO, p. 181-220, 1985.

MARCELO, C. Innovación educativa, asesoramiento y desarrollo profesional. Madrid: CIDE, 1997.

MARTÍN, E. La intervención psicopedagógica en el ámbito de gestión del MEC: la historia de un difícil pero imprescindible factor de calidad. Infancia y Aprendizaje, 87, p. 27-45, 1999.

MONEREO, C.; POZO, J. I. (Coords.). A prática de assessoramento educacional. São Paulo: Artmed, 2007. 
Una mirada crítica a la función asesora desde los servicios de apoyo externo...

MORENO, J. M. Desarrollo del currículum, renovación pedagógica y asesoramiento. In: ESCUDERO, J. M (Ed.). Diseño, desarrollo e innovación del currículum. Madrid: Síntesis, p. 237-263, 1999.

NIETO, J. M. Reconstruir el asesoramiento pedagógico como práctica de apoyo a los centros escolares. Revista de Educación, 311, p. 217-234, 1996.

NIETO, J. M.; BOTÍAS, F. Los equipos de orientación educativa y psicopedagógica. El asesoramiento a centros escolares desde un análisis institucional. Barcelona: Ariel, 2000.

STOLL, L. FINK, D. Para cambiar nuestras escuelas: reunir la eficacia y la mejora. Barcelona: Octaedro, 1999.

V.AA. Repensar el asesoramiento en educación: ¿Qué prácticas para los nuevos retos? Profesorado, revista de currículum y formación del profesorado. Disponible en http://www.ugr.es/local/recfpro/Rev121.html. Acesso en: 20 abr. 2008.

Encaminhado em: 08/07

Aceito em: 03/08 